\title{
Optimal Utilization of Hopfield Neural Network to Resolve Economic Load Dispatch Problem with Wind Penetration
}

\author{
Sankalpa Bohidar, Renu Sharma
}

\begin{abstract}
Basic economic dispatch can be mathematically defined as minimization if the absolute cost of electricity for all plants which are susceptible to limitations. The ELD issue includes a number of distinct concerns. Economic load dispatching shall organize supplies to plants at a minimum price while complying with the limitations or requirements. Wind energy is the rapidly increasing renewable energy source due to its presence in existence and the development of its technology. In this paper, a neuron-based Hopfield neural network is suggested with some modifications to enhance its performance that can be utilized to fix the optimization issue and to resolve the economic dispatch issue by utilizing an energy function including mismatch power, average fuel cost and associated weights. The stabilization assessment of the suggested model is carried out in this paper. The primary reason for the utilization of the Hopfield network is to conduct an accurate selection of weights and features. The usefulness of the suggested network is assessed on economic load dispatch.
\end{abstract}

Keywords: economic load dispatch, wind penetration, Hopfield neural network.

\section{INTRODUCTION}

In recent decades, the implementation of renewable sources of energy for thermal generation has gained a lot of attention from researchers. This is due to the depletion of fossil fuels, along with the upgrade in prices of fuel and issues which are related to thermal fuels. Economic dispatch (ED) is a popular challenge associated with optimization. It mainly focuses on finding a suitable combination of power shares to minimize costing. The economic dispatch issues in basically power systems are computed as stochastic optimization issues associated with uncertainties like forecasting error, market price and alike.

The economic function of the power system includes the solution of two dissimilar issues. The first issue is the commitment unit and second issue is the economic dispatch.The economic dispatch issue (ED) of the energy scheme is to evaluate the optimum mix of energy inputs for all generators that reduces the complete expense of electricity while fulfilling the limitations. Traditional techniques for fixing ED issues are "Lambda iteration" technique, reasonable gradient method, etc. The major downside of all these techniques is that the dimension of the power systems increases, the necessary computational time

\footnotetext{
Revised Manuscript Received on 14 August, 2019.

SankalpaBohidar, Department of Electrical Engineering,Institute of Technical Education and Research,Siksha 'O' Anusandhan(Deemed to be University),Bhubaneswar, Odisha, India.

Renu Sharma, Department of Electrical Engineering,Institute of Technical Education and Research,Siksha 'O' Anusandhan(Deemed to be
} University),Bhubaneswar, Odisha, India.(Email: renusharma@soa.ac.in) period also increases; these techniques cannot be utilized for on-line procedure.

In the latest years, the strategy of the Hopfield Neural Network has been utilized to fix the issue of the economic dispatch of the energy scheme. In this strategy, the "objective function" of the ED issue, with the limitations, is transferred to the' E' energy function, called the "Hopfield Energy function". This energy function would then be reduced by the "Hopfield dynamics" via the iterative method. Standard Hopfield model, used to fix the ED issue, is a constant and probabilistic model along with a "sigmoid function" to define the input/output connection with neurons.

However, the aforementioned model has many drawbacks. The computational time period needed is large in size. The reason for this is the iterative nature of the Hopfield neural network. The production of values associated with dispatch obtained may be inaccurate due to the occurrence of "saturation phenomena" happening in "sigmoid function". Moreover, the factors associated with weights are selected by method of hit and trial.

The most significant issue in the planning and implementation of the electrical energy supply scheme is the efficient scheduling of all batteries in the scheme to fulfill the necessary requirement.In any field, the calculation of intelligence was utilized for the growth of computer technology. The standard economic dispatch is to plan the engaged inputs to satisfy load requirements at minimum working costs while fulfilling all limitations on device.

The primary goal of the ELD issue is to mitigate operating costs by fulfilling the multiple operating limitations in an attempt to meet the load requirement. Several other conventional techniques are used to optimize ELD difficulties, but even in those techniques the actual "cost curves" of the respective units are expected to be linearly growing linear functions in a piecewise method, but the useful schemes are non-linear.

In recent years, standard Hopfield neural network [1], [2]design is the most frequently utilized framework for selfassociation and optimization. It was also utilized for resolving the Economic dispatch issue of power systems. The Hopfield network is a definite kind of recurrent artificial neural network. Generally, the Hopfield networks are coupled with the thoughtof manipulating a human memory with the help of pattern detection and storage technique.Hopfield networks are auto-associations wherein 


\section{OPTIMAL UTILIZATION OF HOPFIELD NEURAL NETWORK TO RESOLVE ECONOMIC LOAD DISPATCH PROBLEM WITH WIND PENETRATION}

node values are incrementally upgraded to the "local computing principle": the new state for each node varies only on its net weighted input at a particular time. The economic dispatch problem is converted into an "energy function E" known as "Hopfield Energy function". This "energy function" is then reduced by the help of iterative techniques that belong to Hopfield dynamics.

This infrastructure is fully linked to the system and the determination of the weight matrix is one of the significant functions for definite applications.In the Hopfield neural network framework, processing tools are called neurons. Each neuron is linked to another neuron by the weight function" $w_{i j}$. In Hopfield's fundamental framework there is no self-connection and the weights are present in a symmetrical way, i.e. " $w_{i j}=w_{j i}$ ". The sustainability of the structure is proved by the power function. The modification of the "Hopfield mode" creates a monotonous decrease in the energy level. As far as optimization is concerned, the "Hopfield neural network" has a well-proven capacity to find alternatives to challenging optimization problems. Researchers used the "Hopfield neural network" to address analog to digital transformation, quadratic task problems, work schedule. In addition to these apps, "Hopfield neural networks" has been used in a number of other applications, such as parametric dynamic system identification, financial charge shipment, and concurrent linear equation resolution.

This research paper is divided into several sections, where chapter II explains about the related work, chapter III presents the proposed work, chapter IV explains about the experimentations done to prove the efficiency of the proposed work and chapter $\mathrm{V}$ concludes all the work done in this research paper.

\section{RELATED WORK}

The application of ANN to multiple Power System problems has risen significantly over the last century. The issue of economic dispatch has been resolved by the use of the "Hopfield Neural Network design".A paper \{\} presented the depiction of major figures in neural networks and their implementation to the economical loading of electrical power. This research reflects one big amount by one neuron, lastly converging to any true value by employing the Hopfield Network. The economic load dispatchauthentication was plotted in the suggested model and checked for four node systems providing " 30,000 MW" of power. Another paper [] expressed Economic Load Dispatch to "Piecewise Quadratic Cost Function" by implementing the Hopfield Neural Network. EDP has been linked to Hopfield cellular network with variable synchronous conversion structure and altered sigmoidal feature. The suggested technique has been screened for issues with "Convex and Non-convex economic dispatch".One researcher submitted "Real Power Optimization with Load Flow" by execution of an Adaptive Hopfield Neural Network. In its place of utilizing the distinctive"B-coefficient technique", the real charge stream is used to calculate the transmitting delay correctly. The paper[3] claims that a comparative analysis of the experiment performance outcomes obtained by using conservative "Newton Raphson" and 'Lagrangianmultiplier" models proposed Genetic Algorithms which at last demonstrates that Genetic Algorithm dispensesimprovedsolutions. A novel PSO technique coupled with an ELD issue to address the environmental radioactivity of the thermal power plant with energy stabilization and transmission limitations is proposed in paper [4].Paper [5] describes the excellent impression of device engagement and economic load dispatch problems for the application of a secure and economical energy scheme.Paper[6] presented a fresh hybrid approach recognized as the Efficient Hybrid Simulated Annealing method. Some researches show that the processes for deviating energy structure stabilizers of fuzzy logic by participating in genetic algorithms[7].Another paper[8]-[11] presents the application of computing "astuteness methods" to the issues of economic load dispatch.Papers[12], [13] describes biogeography-based optimization to clarify economic load dispatch issues of energy plant heat generators.The paper [14] explores the limitations of equality in the equilibrium of energy and the limitations of inequality in generator capability andissues of " $\mathrm{HNN}$ " to clarify the issue of economic environmental dispatch.Paper[15]includes processes for an accurate altered Evolutionary Algorithm. Many other papers[16]-[19]discloses the implementation of fuzzy-logic controllers to overcome the economic dispatch issue of the thermal generation plant.Another paper [20]presented"Hopfield Modeling Framework for Economic Dispatch". The weighting variables connected with the "energy function" that can be either properly chosen or immediately measured in the suggested model. The suggested technique has been evaluated on the "3-bus" and "13-bus" systems. Paper [21], [22] presented the "Hopfield Model to Economic Dispatch with Special Units". This research paper introduces the Hopfield model with three approaches to fix economic dispatch (ED) issues with forbidden working areas. The application of the suggested strategy has been proved by the use of a 15-unit scheme with 4 batteries possessing in forbidden areas. Paper [23], [24]presented "Constraint Economic Dispatch with Prohibited Operating Zones: The Hopfield Neural Network Approach". A fresh classification procedure has been utilized and a computing mechanism for acquiring weights and biases is defined utilizing a loose varying approach for managing limitations. The suggested method has been proved for the " 18 -unit" scheme with 4 batteries present in forbidden areas.

Another paper provides an overview of the algorithms suggested for the" implementation of the Hopfield Neural Network to the issue of the Economic Load Dispatch". Paper 1 portrayed the "Modified Hopfield Network "wherein the internal structures of the neural network are figured by means of a "valid-subspace technique" that ensures conjunction with the equilibrated points that deliver a clarification to the ED problem. Simulation findings and a comparison assessment incorporating a 3-bus sample scheme have been provided to demonstrate the effectiveness of the suggested strategy.

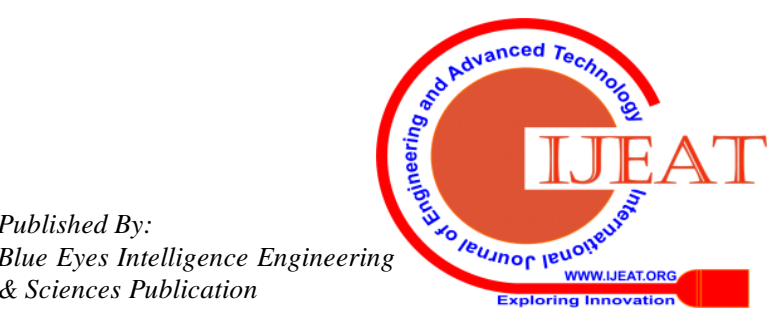




\section{PROPOSED WORK}

This paper introduces and fixes two economic load dispatch designs that are restricted by the probabilistic matrix[25]-[30]. The first model, (ELD.EQ), has a closedended alternative. The second model, (ELD.INEQ), contains more limitations and has no closed-form solution.

The suggested model will enhance a set of constraints:

$$
0 \leq W_{\mathrm{j}} \leq \mathrm{w}_{\mathrm{jr},}(\mathrm{j}=1,2 \ldots \ldots, \mathrm{m})
$$

And swap with the equation below:

$$
\sum_{\mathrm{i}=1}^{\mathrm{n}} \mathrm{x}_{\mathrm{i}}=\mathrm{P}_{\mathrm{d}}+\mathrm{P}_{\mathrm{s}}
$$

So the equation becomes:

$$
\sum_{\mathrm{i}=1}^{\mathrm{n}} \mathrm{x}_{\mathrm{i}}+\Omega(\mathrm{W})=\mathrm{P}_{\mathrm{d}}+\mathrm{P}_{\mathrm{s}}
$$

The energy function for addressing the issue of economic load dispatch with a quadratic cost function is described below:

$$
E=\frac{A\left(P_{D}+P_{L}-\sum x_{i}\right)^{2}}{2}+\frac{B \sum\left(q_{i 0}+q_{i 1} x_{i}+q_{i 2} x_{i}^{2}\right)}{2}
$$

Wherein A, B are the factor associated with weight.

The equation associated with the weights and biases are described below:

$$
\begin{gathered}
T_{i i}=-A-B q_{i 2} \\
T_{i j}=-A \\
I_{i}=A\left(P_{D}+P_{L}\right)-\frac{B q_{i 1}}{2}
\end{gathered}
$$

With all these weights and biases aforementioned, "Hopfield neural network" is simulated for resolving economic load dispatch issue.

This paper discloses the standard Lagrange multiplier method to fix the "ELD EQ". First, there is a construction of the enhanced function:

$$
L\left(x_{1} \lambda\right)=\sum_{i=1}^{n}\left(a_{i 0}+a_{i 1} x_{i}+a_{i 2} x_{i}^{2}\right)+\lambda\left(P_{d}-w_{r} h_{\eta}-\sum_{i=1}^{n} x_{i}\right)
$$

The partial derivativeis:

$$
\begin{gathered}
\frac{\partial L}{\partial x_{j}}=q_{j 1}+2 q_{j 2} x_{j}-\lambda \\
(\mathrm{j}=1,2, \ldots \ldots \ldots \mathrm{n}) .
\end{gathered}
$$

Let,

$$
\frac{\partial L}{\partial x_{j}}=0
$$

Then,

$$
\lambda=\frac{2\left(P_{D}-w_{r} h_{p}\right)+\sum_{i=1}^{n}\left(\frac{q_{i 1}}{q_{i 2}}\right)}{\sum_{i=1}^{n} \frac{1}{q_{i 2}}}
$$

Then after the substitution, the final solution is :

$$
x_{o p t m, j}=\frac{2\left(P_{d}-w_{r} h_{p}\right)+\sum_{i=1}^{n} \frac{q_{i 1}}{q_{i 2}}}{2 q_{j 2} \sum_{i=1}^{n} \frac{1}{q_{i 2}}-\frac{q_{j 1}}{2 q_{j 2}}}
$$

\section{RESULTS AND DISCUSSIONS}

This paper concentrates on the "Hopfield neural network" adhered to the economic load dispatch issue of wind penetration. The model is being evaluated on a six-unit generation scheme, refer to Figure 1. Parameters "K" and "PA" are chosen for the highest convergence characteristics. Here $(\mathrm{k}=1.2,1.7,2.2)$ and $(\mathrm{pa}=0.3,0.4,0.5)$ are considered in this paper. The average loss during the transmission process is neglected during the stimulation. Neuron input is provided as a weighted amount of neurons to other neurons. The penetrating rate is calculated. The best possible valuation of the cost is calculated. It has been noted that the cost function scales linearly after a number of iterations. The ideal cost reduces with the rise in the shape factor $(\mathrm{k})$.

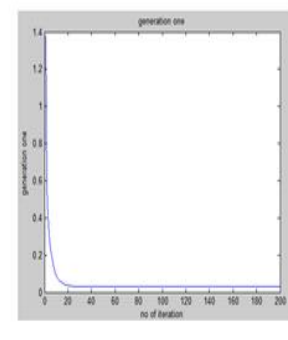

1

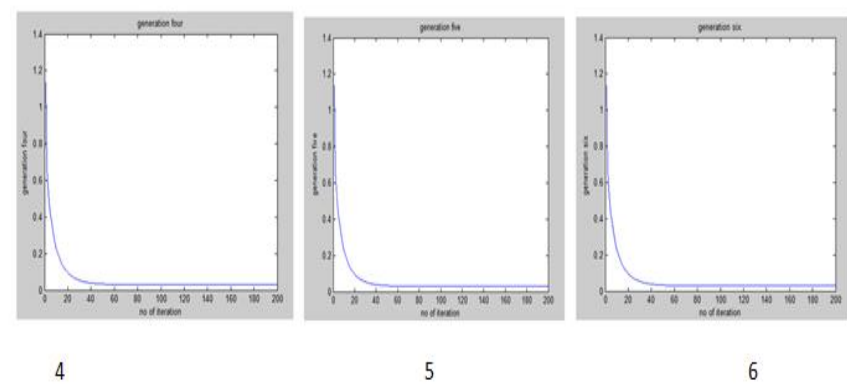

Figure 1 Simulation Result for 6 generations

\section{CONCLUSION}

In this paper, the fast Hopfield neural network is suggested for an economic load dispatch issue with the consumption of wind power including loses related to transmission. In this framework, the probability of stochastic wind power is included as a constraint. This strategy prevents the probability of infeasibility induced by the invasion of the wind. The economic load dispatch issue is resolved for " 6 " unit generating framework by utilizingthe "Hopfield neural network". The threshold parameter "PA" and the form variables for wind speed " $\mathrm{K}$ " are brought into the constraint. Optimal cost reduces with rising "PA" and "K". An energy function that includes the constraints that are imposed is suggested in this paper.

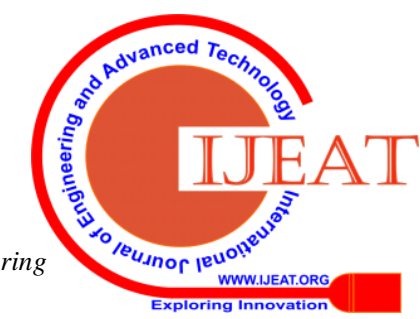




\section{REFERENCES}

1. J. Hertz, A. Krogh, R. G. Palmer, J. Hertz, A. Krogh, and R. G. Palmer, "The Hopfield Model," in Introduction to the Theory of Neural Computation, 2018.

2. A. Barra, A. Bernacchia, E. Santucci, and P. Contucci, "On the equivalence of Hopfield networks and Boltzmann Machines," Neural Networks, 2012.

3. S. Kumar Mishra and S. Kumar Mishra, "A comparative study of solution of economic load dispatch problem in power systems in the environmental perspective," in Procedia Computer Science, 2015.

4. N. S. Singh, J. Catazaro, M. Bernier, R. Powers, and I. Wainer, "Abstract 1172: (R,R')-4'-Methoxy-1naphthylfenoterol decreases glycolytic activity in the PANC-1 pancreatic cancer cell line," 2015.

5. P. Surekha and S. Sumathi, "Solving economic load dispatch problems using differential evolution with opposition based learning," WSEAS Trans. Inf. Sci. Appl., 2012.

6. M. Vanitha and K. Thanushkodi, "Solving non-convex economic load dispatch problem by efficient hybrid simulated annealing algorithm," in Proceedings of 2012 IEEE International Conference on Advanced Communication Control and Computing Technologies, ICACCCT 2012, 2012.

7. M. Dubey and A. Dubey, "Genetic algorithm based design of fuzzy logic power system stabilizers in multimachine power system," World Acad. Sci. Eng. Technol., vol. 42, pp. 961-968, 2010.

8. K. Borana, "Genetic algorithms and its application to economic load dispatch," in AIP Conference Proceedings, 2010, vol. 1324, pp. 239-243.

9. G. Kannan and N. Karthik, "Application of fireflies algorithm to solve economic load dispatch," in Proceeding of the IEEE International Conference on Green Computing, Communication and Electrical Engineering, ICGCCEE 2014, 2014.

10. "Economic Load Dispatch," 2018, pp. 46-64.

11. J. A. Laghari, H. Mokhlis, A. H. A. Bakar, and H. Mohamad, "Application of computational intelligence techniques for load shedding in power systems: A review," Energy Convers. Manag., vol. 75, pp. 130-140, 2013.

12. S. Mondal, A. Bhattacharya, and S. H. Nee Dey, "Multiobjective economic emission load dispatch solution using gravitational search algorithm and considering wind power penetration," Int. J. Electr. Power Energy Syst., 2013.

13. E. Arriagada, E. López, M. López, R. Blasco-Gimenez, C. Roa, and M. Poloujadoff, "A probabilistic economic dispatch model and methodology considering renewable energy, demand and generator uncertainties," Electr. Power Syst. Res., 2015.

14. R. Bouakacha and L. Salah, "Economic dispatch by the combination of HNN and the Lagrange method," in ELECO 2013 - 8th International Conference on Electrical and Electronics Engineering, 2013.

15. Q. Zhang, D. Zou, N. Duan, and X. Shen, "An adaptive differential evolutionary algorithm incorporating multiple mutation strategies for the economic load dispatch problem," Appl. Soft Comput. J., 2019.

16. S. Al-Sakkaf, M. Kassas, M. Khalid, and M. A. Abido, "An energy management system for residential autonomous DC microgrid using optimized fuzzy logic controller considering economic dispatch," Energies, 2019.

17. S. Vijayaraj and R. K. Santhi, "MULTI-AREA ECONOMIC DISPATCH WITH PROHIBITED OPERATING ZONES USING IMPROVED BAT ALGORITHM," Int. J. Multidiscip. Res. Mod. Educ. ISSN Vol. II, Issue II, 2016.
18. H. Nezamabadi-Pour, S. Yazdani, M. M. Farsangi, and M. Neyestani, "A solution to an economic dispatch problem by a fuzzy adaptive genetic algorithm," Iran. $J$. Fuzzy Syst., 2011.

19. E. R. Sanseverino, M. L. Di Silvestre, G. Zizzo, R. Gallea, and N. N. Quang, "A Self-Adapting approach for forecast-Less scheduling of electrical energy storage systems in a liberalized energy market," Energies, 2013.

20. V. N. Dieu, P. Schegner, and W. Ongsakul, "Augmented Lagrange Hopfield network for economic dispatch problems," in AIP Conference Proceedings, 2012, vol. 1499, pp. 36-45.

21. F. N. Al Farsi, M. H. Albadi, N. Hosseinzadeh, and A. H. Al Badi, "Economic Dispatch in power systems," in 2015 IEEE 8th GCC Conference and Exhibition, GCCCE 2015, 2015.

22. I. R. Fitri, J. S. Kim, and H. Song, "Distributed economic dispatch for power systems," in 2017 Asian Control Conference, ASCC 2017, 2018, vol. 2018-January, pp. 2808-2812.

23. F. Benhamida, A. Bendaoud, and A. Ayad, "A deterministic hopfield model to dynamic economic dispatch with ramp limit and prohibited zones," Istanbul Univ. - J. Electr. Electron. Eng., vol. 12, no. 1, pp. 1465 1471, 2012.

24. [24] F. Benhamida, A. Bendaoued, K. Medles, A. Ayad, and A. Tilmatine, "A solution to dynamic economic dispatch with prohibited zones using a Hopfield neural network," in ELECO 2011 - 7th International Conference on Electrical and Electronics Engineering, 2011.

25. Z. Weng, L. Shi, Z. Xu, Q. Lu, Y. Ni, and L. Yao, "Power system dynamic economic dispatch incorporating wind power cost," Zhongguo Dianji Gongcheng Xuebao/Proceedings Chinese Soc. Electr. Eng., vol. 34, no. 4, pp. 514-523, 2014.

26. A. Naresh Kumar and D. Suchitra, "AI based Economic Load Dispatch incorporating wind power penetration," in Proceedings of the 2011 International Conference on Electrical Engineering and Informatics, ICEEI 2011, 2011.

27. T. S. Zhan, C. C. Kao, and B. R. Niu, "Dynamic economic dispatch incorporating wind power generation with carbon trading," in Lecture Notes in Electrical Engineering, 2014, vol. 293, pp. 163-170.

28. J. Meng, G. Y. Li, and S. J. Cheng, "Economic dispatch for power generation system incorporating wind and photovoltaic power," in Applied Mechanics and Materials, 2014, vol. 441, pp. 263-267.

29. S. Tong, Y. Zhang, and J. Qi, "Impact of electric vehicles as interruptible load on economic dispatch incorporating wind power," in IET Conference Publications, 2012, vol. 2012, no. $611 \mathrm{CP}$.

30. M. G. Dozein, J. Ansari, and M. Kalantar, "Economic Dispatch incorporating wind power plant using Particle Swarm Optimization," in 2012 2nd Iranian Conference on Renewable Energy and Distributed Generation, ICREDG 2012, 2012, pp. 178-182. 